DOI 10.21292/2078-5658-2016-13-3-63-70

\title{
АТИПИЧНЫЙ ГЕМОЛИТИКО-УРЕМИЧЕСКИЙ СИНДРОМ У ПАЦИЕНТКИ С МЕНОМЕТРОРРАГИЕЙ (клиническое наблюдение)
}

\author{
А. В. ПЫРЕГОВ, Т. А. ФЕДОРОВА, А. Ю. КОРОЛЕВ, К. И. ГРИЩУК
}

ФГБУ «Научный центр акушерства, гинекологии и перинатологии им. акад. В. И. Кулакова» МЗ РФ, Москва

В статье приведено описание клинического наблюдения развития атипичного гемолитико-уремического синдрома у пациентки с менометроррагией, манифестировавшего на фоне маточного кровотечения и сопровождавшегося развитием острой почечной недостаточности. Ключевые слова: атипичный гемолитико-уремический синдром, острая почечная недостаточность, гемодиализ с ультрафильтрацией, экулизумаб.

\section{ATYPICAL HEMOLYTIC-UREMIC SYNDROME IN THE FEMALE PATIENT WITH CONCURRENT MENOMETRORRHAGIA (clinical case)}

\section{A. V. PYREGOV, T. A. FEDOROVA, A. YU. KOROLEV, K. I. GRISCHUK}

Research Center for Obstetrics, Gynecology and Perinatology, Moscow, Russia

The article describes the follow-up over the development of atypical hemolytic-uremic syndrome in the female patient suffering menometrorrhagia which manifested with concurrent metrorrhagia and was accompanied with acute renal failure.

Key words: atypical hemolytic-uremic syndrome, acute renal failure, hemodialysis with ultra-filtration, eculizumab.

Атипичный гемолитико-уремический синдром (аГУС), наряду с типичным ГУС и тромботической тромбоцитопенической пурпурой (ТТП), является классическим заболеванием из группы тромботических микроангиопатий (ТМА). Сегодня аГУС рассматривается как хроническое системное заболевание генетической природы, в основе которого лежит неконтролируемая активация альтернативного пути комплемента, ведущая к генерализованному тромбообразованию в сосудах микроциркуляторного русла (комплемент-опосредованная ТМА) $[1,8,29]$. Морфологически ТМА рассматривается как особый тип повреждения сосудов, сопровождающийся отеком эндотелиальных клеток с их отслойкой от базальной мембраны, расширением субэндотелиального пространства с накоплением в нем аморфного мембраноподобного материала и образованием тромбов, содержащих тромбоциты и фибрин, что приводит к окклюзии просвета сосуда, вызывая развитие ишемии органов и тканей. TMA всегда сопровождается тромбоцитопенией, развивающейся вследствие потребления тромбоцитов в процессах распространенного тромбообразования, микроангиопатической гемолитической анемией, лихорадкой, поражением почек, печени, дыхательной и центральной нервной системы [1, 15]; аГУС относится к редким заболеваниям, его частота составляет 2-7 случаев на 1000 000, чаще до $60 \%$ заболевших - дети, в старшем возрасте чаще страдают женщины $[1,28]$. Этиология обусловлена мутациями генов регуляторных белков и компонентов комплемента, мембранного кофакторного протеина, тромбомодулина, CFH, CFI, CFB (факторы H, I, В) либо наличием антител к СFН. Эти факторы приводят к развитию хронической неконтро- лируемой активации комплемента и развитию комплемент-зависимой ТМА $[8,17,33]$. Прогноз неблагоприятный со смертностью до $25 \%$ и с развитием почечной недостаточности в $56 \%$ случаев [15, 22, 30, 39]. У половины выживших пациентов функция почек полностью не восстанавливается. Без семейного анамнеза аГУС рассматривается как спорадический и развивается при наличии других триггерных факторов, таких как системные аутоиммунные заболевания (системная красная волчанка, антифосфолипидный синдром, системная склеродермия, ревматоидный артрит и др.), инфекции, кровотечения, онкологические заболевания, лекарственные препараты (антибиотики, тиклопидины, оральные контрацептивы) [11, 21, 34]. Обычно заболевание развивается внезапно, может сопровождаться астеновегетативным синдромом, тошнотой, рвотой, затрудненным дыханием, отеками. В 20\% случаев возможны симптомы угнетения сознания и судороги вследствие кровоизлияния, отека мозга. При лабораторном исследовании выявляется характерная диагностическая триада аГУС: тромбоцитопения, почечная дисфункция (повышение уровня мочевины и креатинина) с развитием олигоурии/анурии и протеинурии, внутрисосудистый гемолиз: повышение ЛДГ, появление клеток шизоцитов $[22,34]$. Для исключения ТТП всем больным с ТМА необходимо также определение активности ADAMTS-13. Активность ADAMTS-13 в норме составляет $80-110 \%$. Снижение активности ADAMTS-13 до 5\% и менее свидетельствует в пользу диагноза ТТП. У пациентов с аГУС активность ADAMTS-13 всегда превышает 5\%. Следует подчеркнуть, что определение активности ADAMTS-13 необходимо проводить до назначения 
плазмотерапии, при которой показатели активности ADAMTS-13 будут недостоверными [3, 5, 8, 10, 34]. При лечении аГУС проводят плазмотерапию в виде трансфузий донорской свежезамороженной плазмы или в режиме плазмообмена с удалением 1-1,5 ОЦП и замещением ее свежезамороженной донорской плазмой, которая содержит функционально активные регуляторные белки системы комплемента, что устраняет дефицит собственных естественных регуляторов - факторов Н, I, В. Кроме того, донорская плазма - сбалансированный источник естественных компонентов плазмы, обладающих протеолитической активностью в отношении сверхкрупных мультимеров фактора Виллебранда, антикоагулянтов и компонентов системы фибринолиза, что приводит к уменьшению процессов тромбообразования в микроциркуляторном русле. При плазмообмене не возникает таких осложнений, как гиперволемия и гипергидратация, что особенно важно у пациентов с олигурией, сердечно-сосудистыми нарушениями. При экстракорпоральной гемокоррекции происходит удаление крупнодисперсных белков, циркулирующих иммунных комплексов, эндогенных циркулирующих ингибиторов комплемента и циркулирующих антител к фактору Н. Считается, что процедуры плазмообмена следует продолжить до нормализации числа тромбоцитов, прекращения гемолиза и улучшения функции почек, поэтому необходимо контролировать количество тромбоцитов, гемоглобина, шизоцитов, уровня ЛДГ [34]. Пациентам с аГУС с выраженной анемией (уровень гемоглобина менее 70-75 г/л) показаны трансфузии эритроцитных донорских компонентов (эритровзвеси, отмытых эритроцитов). Также проводится симптоматическая терапия (при артериальной гипертензии назначение антигипертензивных препаратов, низкомолекулярных гепаринов, антибактериальная терапия, глюкокортикоидные препараты при лечении системной красной волчанки и других сопровождающих аГУС заболеваний). В последние годы в случаях неэффективности проводимой терапии при рецидивирующем течении заболевания назначают экулизумаб - препарат группы комплемент-ингибирующих антител (человеческие моноклональные антитела к С5-фактору комплемента). Данный препарат блокирует расщепление C5 на C5a и C5b, что препятствует образованию мембраноатакующего комплекса C5b-9 и подавляет провоспалительное, протромботическое и литическое действия комплемента, предотвращая повреждение эндотелия и прекращая процессы микроциркуляторного тромбообразования, что приводит к обратному развитию ТМА. В разработке находятся растворимые формы ингибитора С3/C5-конвертазы, рецептора комплемента I $[12,16]$.

Клиническое наблюдение. Пациентка А., 50 лет, поступила в ФГБУ «НЦАГиП им. В. И. Кулакова» 13.01.2016 г. с жалобами на кровянистые выделения из половых путей, продолжающиеся в течение 2 нед. В клиническом анализе крови отмечено сни- жение гемоглобина до 72 г/л, количество эритроцитов $-3,15 \times 10^{12} /$ л. Диагноз: дисфункциональное маточное кровотечение; постгеморрагическая анемия; ожирение 3-й степени. В экстренном порядке выполнены гистероскопия, раздельное диагностическое выскабливание слизистой полости матки и цервикального канала в условиях спинальной анестезии. Выбор регионарной методики был сделан в связи с приемом пациенткой пищи менее 2 ч до операции, оценкой верхних дыхательных путей по шкале Mallampati IV, морбидным ожирением 3-й степени. Объем оперативного вмешательства был минимально необходимым для остановки кровотечения. Интраоперационно отмечалась тенденция к гипотензии, что потребовало введения вазопрессоров. По окончании операции с целью сокращения матки внутривенно капельно вводили окситоцин 2,5 Ед, после чего отмечены угнетение сознания на 15 с и снижение артериального давления (АД) до 80/40 мм рт. ст. Инфузия составила 1000 мл сбалансированных кристаллоидных растворов, кровопотеря - 300 мл, диурез - 50 мл. Транспортировку в отделение реанимации и интенсивной терапии (ОРИТ) пациентка перенесла удовлетворительно.

При поступлении в ОРИТ отмечались тошнота и рвота, купированные введением ондасетрона. Отмечалась тахикардия до 115 в минуту. По мочевому катетеру - светлая моча 100 мл. Выполнен общий анализ крови: гемоглобин - 59 г/л, гематокрит - 19\%, эритроциты - 2,69 × 10²/л, тромбоциты - $183 \times 10^{9} /$ л. Пациентке проведена гемотрансфузия в объеме 670 мл отмытых эритроцитов. Через 1 ч была отмечена тенденция к гипертензии (систолическое АД - 150-160 мм рт. ст.). Начата антигипертензивная терапия (ингибитор ангиотензинпревращающего фермента - каптоприл и блокатор кальциевых каналов - нифедипин). $\mathrm{SpO}_{2}$ составляла 93-95\% с инсуффляцией кислорода. Через 3 ч после трансфузии отмечено снижение темпа диуреза, введен фуросемид 10 мг с эффектом. В клиническом анализе крови: гемоглобин - 79 г/л, гематокрит - 24\%, эритроциты - 3,48 × $10^{12} /$ л, тромбоциты - $64 \times 10^{9} /$ л. Суточный диурез составил 570 мл.

На 2-е сут состояние пациентки с отрицательной динамикой, отмечены жалобы на слабость, тошноту. Для уточнения диагноза в ОРИТ пациентке выполнен ряд лабораторных исследований, ЭКГ, Эхо-КГ, УЗИ брюшной полости, УЗИ вен нижних конечностей, фиброэзофагогастродуоденоскопия. В клиническом анализе крови - признаки гипохромной анемии (гемоглобин - 79 г/л), тромбоцитопения $\left(64 \times 10^{9} /\right.$ л), нейтрофильный лейкоцитоз (лейкоциты $\left.-16,6 \times 10^{9} / л\right)$. В коагулограмме - признаки хронической гиперкоагуляции, выраженная тромбинемия без явных признаков потребления (активированное частичное тромбопластиновое время (АЧТВ) - 19 с, D-димер - 9999 мкг/л, активность AT-III - 103\%, фибриноген - 4 г/л, r + k - 16 c). 
Общий анализ мочи - протеинурия, глюкозурия. После дуплексного сканирования выявлен массивный тромбоз глубоких вен нижних конечностей (окклюзивный тромбоз задней большеберцовой вены (ЗББВ) в средней трети, полностью суральные вены правой голени, ЗББВ в верхней и средней трети и суральные вены полностью в левой голени). По данным Эхо-КГ: умеренная легочная гипертензия (СДЛА 60 мм рт. ст.), глобальная сократимость левого желудочка в пределах нормы. Центральное венозное давление (ЦВД) - 26-28 см вод. ст. Выставлен диагноз: состояние после гистероскопии, раздельного диагностического выскабливания, постгеморрагическая анемия тяжелой степени, тромбоз глубоких вен нижних конечностей, морбидное ожирение 3-й степени, гипертоническая болезнь 2-й стадии, хронический панкреатит, хронический гастрит, состояние после холецистэктомии, тромбоэмболия легочной артерии(?). В связи с развившейся острой почечной недостаточностью от введения контрастных веществ для проведения КТ-ангиографии решено воздержаться. Начаты терапия низкомолекулярными гепаринами в лечебных дозах, профилактика стресс-язв, стимуляция диуреза до 1200 мг фуросемида без ответа. Продолжена терапия артериальной гипертензии, кислородотерапия $\left(\mathrm{SpO}_{2}-92-96 \%\right)$. К концу 2 -х сут сохранялась тяжелая анемия (гемоглобин - 68 г/л), тромбоцитопения $\left(28 \times 10^{9} / л\right)$, лейкоцитоз, отмечено появление шизоцитов. В биохимическом анализе крови - гипопротеинемия (общий белок - 51 г/л, альбумин - 29 г/л), гипергликемия (10 ммоль/л), отмечено нарастание содержания мочевины (14,1 ммоль/л) и креатинина (250 мкмоль/л), повышение уровня ЛДГ до 600 Ед/л. Несмотря на стимуляцию фуросемидом, суточный объем мочи составил 200 мл. Гидробаланс за сутки составил +2600 мл. Для дифференциальной диагностики между ТТП, аГУС и антифосфолипидным синдромом пациентке выполнено исследование активности ADAMTS-13 (дезинтегрин металлопротеиназы тромбоспондина 1 типа 13), уровня антифосфолипидных антител, волчаночного антикоагулянта для исключения диагноза антифосфолипидного синдрома и системной красной волчанки. В связи с развитием почечной недостаточности проведена процедура гемодиафильтрации, объем ультрафильтрата составил 1100 мл.

На 3-и сут пребывания в ОРИТ - состояние без отрицательной динамики. Сохранялся астеновегетативный синдром. Пациентке катетеризировали правую внутреннюю яремную вену. Проведены коррекция гипоальбуминемии (200 мл 20\% альбумина) и коррекция анемии (500 мл эритроцитарной взвеси). К клиническому диагнозу добавлены: острая почечная недостаточность (RIFLE 4-5 ст.); сахарный диабет 2-го типа, впервые выявленный. Осуществлена замена эноксапарина гепарином 25000 Ед в сутки. Инфузию гепарина проводили с коррекцией скорости в зависимости от значения
АЧТВ. Контроль АЧТВ осуществляли каждые 4 ч, целевым уровнем АЧТВ было 90 с. Отмечалось повышение содержания мочевины до 16,9 ммоль/л и креатинина до 404,9 мкмоль/л, уровня D-димера до 4567 мкг/л, глюкозы до 8,5 ммоль/л. В плане антигипертензивной терапии выбор сделан в пользу блокаторов медленных кальциевых каналов ввиду их минимального влияния на почечную функцию. Диурез за сутки составил 370 мл, со стимуляцией петлевыми диуретиками 1000 мг за сутки. ЦВД колебалось в пределах 18-23 см вод. ст. Кислородотерапию продолжали ( $\left.\mathrm{SpO}_{2}-90-93 \%\right)$. Проводили процедуру гемодиафильтрации с объемом ультрафильтрата 1000 мл.

На 4-е сут состояние оставалось тяжелым, без ухудшения. Антикоагулянтная терапия продолжена с целевым значением АЧТВ 90-100 с (28 000 Ед нефракционированного гепарина за сутки). По лабораторным данным: сохранялись анемия (гемоглобин - 71 г/л), тромбоцитопения $\left(41 \times 10^{9} / л\right)$, нейтрофильный лейкоцитоз, шизоциты - 50 на 10 000; умеренная гипопротеинемия, азотемия (мочевина - 19 ммоль/л, креатинин 460 мкмоль/л), уровень электролитов в норме. Уровень глюкозы находился в пределах 8-10 ммоль/л. АД скорректировано до 120/80 мм рт. ст., $\mathrm{SpO}_{2}$ составляла 96-98\% с инсуффляцией кислорода. Диурез за сутки составил 600 мл со стимуляцией фуросемидом 1000 мг, баланс жидкости +1000 мл. ЦВД колебалось в пределах 15-20 см вод. ст.

В последующем отмечалось увеличение уровня креатинина до 630 мкмоль/л, мочевины до 29 ммоль/л. Отмечено повышение лактатдегидрогеназы до 2 500. Гликемия оставалась в пределах 7-9 ммоль/л. Диурез за сутки - 1340 мл. Учитывая выявленный симптомокомплекс ТМА (шизоцитоз - 50 на 10 000, ЛДГ - 2 500, тромбоцитопения $60 \times 10^{9} /$ л), отрицательный волчаночный коагулянт, тромбоз глубоких вен нижних конечностей, уровень ADAMTS-13 выше 10\%, выставлен диагноз: атипичный гемолитико-уремический синдром. После предварительного вакцинирования конъюгированной тетравакциной Менцевакс против Neisseria meningitidis ( так как на фоне применения препарата возрастает риск развития менингита) пациентке введено 900 мг экулизумаба внутривенно.

При дальнейшем наблюдении после ультразвукового сканирования вен нижних конечностей отмечена реканализация всех тромбозов. По лабораторным данным: увеличился уровень тромбоцитов $\left(113 \times 10^{9} /\right.$ л), уровень гемоглобина составил 77 г/л, сохранялись гипопротеинемия (белок 49 г/л), высокие уровни креатинина (427 мкмоль/л) и мочевины (21 моль/л). Уровень D-димера составлял 4500 мкг/л, АЧТВ поддерживался на уровнях от 40 до 60 с. Пациентке проведен третий сеанс гемодиафильтрации с объемом ультрафильтрата 950 мл. Гемодинамика за время наблюдения оставалась стабильной. ЦВД составляло 14-20 см вод. ст. Диурез за сутки со стимуляцией составил 1450 мл. 
Ввиду возможной связи тромбоцитопении с гепаринотерапией нефракционированный гепарин заменен далтепарином натрия в дозировке 15000 Ед в сутки. С целью выяснения причины диспептических явлений выполнена эзофагогастродуоденоскопия (ЭГДС). По результатам ЭГДС выявлены эрозии в антральной части желудка и в луковице двенадцатиперстной кишки. Терапия продолжена в прежнем объеме. По лабораторным данным: сохранялась анемия (гемоглобин - 72 г/л), увеличилось количество тромбоцитов до $177 \times 10^{9} /$ л, нормализовалась лейкоцитарная формула. По биохимическому анализу крови: сохранялась умеренная гипопротеинемия, уровень креатинина 358 мкмоль/л, мочевины - 15 ммоль/л. Диурез за сутки составил 1500 мл со стимуляцией (фуросемид $80 \mathrm{мг) \text {. }}$

На 13-е сут пациентка в удовлетворительном состоянии с диагнозом: атипичный гемолитико-уремический синдром, микроангиопатия, анемия средней степени тяжести, поражение поджелудочной железы, желудочно-кишечного тракта, гипертоническая болезнь 2-й стадии, 3-й степени, риск 3 , сахарный диабет 2-го типа, тромбоз глубоких вен нижних конечностей (реканализация), ожирение 3-й степени переведена в отделение хирургии для дальнейшего лечения.

На 15-е сут повторно внутривенно введено 900 мг экулизумаба, уровень креатинина в динамике снижался от 340 до 104 мкмоль/л. Уровень тромбоцитов увеличился до 430 тыс., гемоглобина - до 76 г/л. По УЗДГ вен нижних конечностей - начальная реканализация. Продолжены терапия низкомолекулярными гепаринами, антибиотикотерапия, гипотензивная терапия. Гистологическое исследование слизистой матки - эндометрий в стадии пролиферации (исключен онкопроцесс). На 21-е сут пациентка была переведена для продолжения терапии в МОНИКИ, где была проведена терапия экулизумабом. В результате терапии отмечено снижение уровня креатинина до 104 мкмоль/л, ЛДГ до 350 Ед/л, тромбоциты $-420 \times 10^{9} /$ л, гемоглобин -105 г/л, что явилось критериями для отмены дальнейшего введения экулизумаба.

\section{Обсуждение}

Таким образом, аГУС - редкое, тяжелое заболевание, сопровождающееся почечной недостаточностью, тромботическими осложнениями, тромбоцитопенией, микроангиопатической гемолитической анемией, нередко с неблагоприятным прогнозом. В США аГУС встречается в 1-2 случаях на 1 млн жителей в год $[9,32]$. Впервые термин аГУС был использован в 1955 г. Гассер описал 5 детей в возрасте от 2 месяцев до 7 лет с приобретенной гемолитической анемией, «необычными пойкилоцитами» в мазке крови и почечной недостаточностью, 3 из которых имели тромбоцитопению; все пациенты умерли [14].
В данной статье представлено клиническое наблюдение пациентки 50 лет с наличием ряда предрасполагающих факторов к развитию аГУС - длительное кровотечение, ожирение, гипертоническая болезнь. Описанное состояние представляет угрозу для жизни и требует немедленной терапии, чтобы избежать необратимого повреждения органов, ведущего к летальному исходу, так как состояния, которые сопровождаются ТМА (аГУС, ТТП, антифосфолипидный синдром), приводят к развитию полиорганной недостаточности и гибели больных [6]. Также ТМА может присутствовать при злокачественных новообразованиях, химиотерапии или при трансплантации костного мозга [2].

Дифференциальная диагностика аГУС в данном случае представлялась достаточно сложной задачей, требовавшей исключения ТТП, КАФС, которые клинически проявляются массивным тромбозом и ишемией органов, а также органной недостаточности [19]. Пациентка имела все признаки наличия ТМА, с поражением сосудов нижних конечностей, почек, поджелудочной железы. Невозможно было полностью исключить и мезентериальный тромбоз, так как развитие почечной недостаточности исключало исследование с применением контрастирования. Важным в дифференциальной диагностике аГУС и ТТП является уровень ADAMTS-13. При ТTП дефицит в плазме ADAMTS-13 приводит к накоплению очень большого количества фактора Виллебранда на эндотелии. В нормальных физиологических условиях ADAMTS-13 расщепляет излишнее количество фактора Виллебранда, освобождая эндотелиальные клетки после повреждения сосудов. Этот процесс имеет решающее значение для управления активностью фактора Виллебранда и для поддержания равновесия гемостаза. Значительное снижение активности ADAMTS-13 (<5-10\%) приводит к агрегации тромбоцитов на клетках эндотелия, активированных фактором Виллебранда, и к образованию микротромбов в небольших артериолах и капилляpax по всему телу [8, 31]. ТТП потенциально может быть точно дифференцирована от аГУС по тяжелому дефициту ADAMTS-13 протеазы. В данном наблюдении активность фермента составляла $23 \%$, следовательно, диагноз ТТП был исключен. Есть многочисленные сообщения, описывающие появления симптомов аГУС после триггерного воздействия окружающей среды. Беременность, лекарства, злокачественные новообразования, заболевания соединительной ткани и некоторые метаболические патологии также являются доказанными триггерами $[1,23,32]$. В этих случаях следует устранить предполагаемый триггер, который инициировал появления симптомов, что может привести к разрешению ТМА. Если ТМА сохраняется, у пациента имеется генетическая предрасположенность к аГУС [3, 35]. Вероятнее всего, у данной пациентки триггерным фактором послужило наличие хронического кровотечения, метаболического синдрома и, возможно, неустановленных факторов в сумме. Хотя диагноз тяжелой 
ADAMTS13-недостаточности (ТTП) может быть почти исключен на основании уровня креатинина в крови выше 150-200 мкмоль/л и тромбоцитов ниже $30 \times 10^{9} /$ л $[8,10,37]$, что тоже имело место у пациентки уже на 2-е сут терапии. Измерение сывороточных уровней C3, C4, CFH и CFI и генетический скрининг мутаций не проводили, хотя они могут быть полезными [3, 36], но следует отметить, что нормальный уровень белков комплемента или отсутствие мутации не исключает диагноз аГУС, так как количество комплемента в плазме не коррелирует с его активностью на поверхности эндотелия $[13,24,26]$.

Основой лечения аГУС является плазмозаместительная терапия с заменой больших объемов с максимально ранним началом. В то время как плазмозамещение первоначально устраняет гематологические симптомы у некоторых пациентов, до 65\% пациентов с аГУС требуют проведения заместительной почечной терапии, так как часто развивается необратимое повреждение почек [4]. АГУС почти всегда протекает с поражением почек, в $72 \%$ случаев требующим проведения гемодиализа, в то время как при дефиците ADAMTS-13 о развитии почечной недостаточности сообщается только в $10 \%$ случаев $[5,20]$. Пациентке в данном наблюдении было проведено три процедуры заместительной почечной терапии с положительным результатом, без дальнейшего формирования хронической почечной недостаточности, но следует помнить, что гематологическое улучшение не означает, что ТМА не продолжается в органах. В рассмотренном случае было решено начать терапию препаратом экулизумабом в связи с тяжестью состояния пациентки, имевшей признаки перегрузки малого круга кровообращения [13]. В 2009 г. группой исследователей был опубликован первый успешный пример лечения аГУС с использованием экулизумаба [4]. В США при постановке диагноза аГУС экулизумаб рекомендуется в качестве первой линии терапии [3]. Препарат представляет собой моноклональное антитело, которое связывается с белком комплемента С5, блокируя образование на клеточной мембране повреждающего комплекса C5b-9. Два перспективных исследования, включивших 37 пациентов с аГУС, показали, что введение экулизумаба дает быстрое и устойчивое торможение процесса ТМА со значительными улучшениями клинических исходов (гемолиз, количество тромбоцитов и почечная функция), ранней отменой плазмозамещающей терапии и снижением необходимости в диализе на 80\% [24]. Пациентке было введено 900 мг экулизумаба после предварительной вакцинации менцеваксом. На следующие сутки после введения препарата отмечено значительное повышение уровня тромбоцитов: с 40 тыс. до 120 тыс., диурез увеличился до 1700 мл, уровень азотемии значительно снизился. Рядом исследователей у больных после лечения экулизумабом было зарегистрировано два случая менингококковой инфекции, которые были купированы при терапии антибиотиками [18].
Из-за механизма действия экулизумаба требуются превентивные меры до начала лечения - прививки против Neisseria Meningitides и при необходимости антибиотикопрофилактика [3]. В нашем клиническом примере введение экулизумаба проведено после предварительной вакцинации менцеваксом. В случае формирования хронической почечной недостаточности может потребоваться трансплантация почки. Однако из-за высоких рисков рецидива ТМА и потери трансплантата аГУС является противопоказанием для донорства живой почки [7, 27]. Перед пересадкой должен быть выполнен полный генетический анализ, чтобы обнаружить мутации и анти-СFH антитела. ТМА после пересадки почки имеет место у $50 \%$ пациентов, отторжение трансплантата происходит в 80-100\% случаев при развитии ТМА [25]. Пациенты, у которых считается возможной пересадка почки, должны быть обследованы и оценены индивидуально для оценки риска отторжения трансплантата [3, 27]. Так как печень является источником некоторых белков комплемента, пересадка и печени, и почек может быть вариантом для профилактики дальнейшей ТМА у больных аГУС. У больных аГУС с нормальной функцией почек изолированная трансплантация печени не рекомендована из-за рисков, связанных с пожизненной иммуносупрессивной терапией (значительно перевешивают риски при долгосрочной терапии моноклональными антителами) [38]. Нашей пациентке была продолжена терапия экулизумабом с положительным эффектом со снижением уровня креатинина, ЛДГ, увеличением уровня тромбоцитов до нормальных значений, гемоглобина, появлением признаков начальной реканализации вен нижних конечностей.

Представленное клиническое наблюдение демонстрирует трудности в диагностике аГУС, необходимость мультидисциплинарного подхода при обследовании и лечении таких пациентов. Возможно успешное лечение аГУС без проведения плазмозаместительной терапии, но при своевременном начале заместительной почечной терапии с введением современного патогенетически обоснованного препарата экулизумаб, что позволяет достаточно быстро компенсировать состояние пациентки, улучшить исход заболевания без формирования хронической почечной недостаточности.

\section{Заключение}

Целью публикации является демонстрация очень редкого случая развития атипичного гемолитико-уремического синдрома в послеоперационном периоде у гинекологической пациентки. Редкая встречаемость данного заболевания обусловливает трудности в своевременной постановке диагноза. Вопрос дифференциальной диагностики между антифосфолипидным синдромом, системной красной волчанкой, ТТП и аГУС является одновременно сложным и важным. Клинико-лабораторные проявления этих заболеваний во многом сходны, одна- 
ко подходы к лечению различаются. Максимально ранние диагностика, включая исследование на дефицит ADAMTS-13 при выявлении тромбоцитопении (до начала плазмотерапии), и начало лечения, в том числе терапия моноклональными антителами и заместительная почечная терапия, являются необходимыми и прогностически благоприятными для пациента.

\section{ЛИТЕРАТУРА}

1. Клинические рекомендации по диагностике и лечению атипичного гемолитико-уремического синдрома // Нефрология и диализ. - 2015. - Т. 17 № 3. http://www.nephro.ru/content/files/242-264.pdf

2. Ariceta G., Besbas N., Johnson S. et al. European Paediatric Study Group for HUS. Guideline for the investigation and initial therapy of diarrhea-negative hemolytic uremic syndrome // Pediatr. Nephrol. - 2009. Vol. 24. - P. 687-696.

3. Besbas N., Karpman D., Landau D. et al. A classification of hemolytic uremic syndrome and thrombotic thrombocytopenic purpura and related disorders // Kidney Int. - 2006. - Vol. 70. - P. 423-431.

4. Campistol J. M., Arias M., Ariceta G. et al. An update for atypical haemolytic uraemic syndrome: diagnosis and treatment. A consensus document // Nefrologia. - 2013. - Vol. 33. - P. 27-45.

5. Caprioli J., Noris M., Brioschi S. et al. International Registry of Recurrent and Familial HUS/TTP. Genetics of HUS: the impact of MCP, CFH, and IF mutations on clinical presentation, response to treatment, and outcome // Blood. - 2006. - Vol. 108. - P. 1267-1279.

6. Cataland S. R., Wu H. M. Atypical hemolytic uremic syndrome and thrombotic thrombocytopenic purpura: clinically differentiating the thrombotic microangiopathies // Eur. J. Intern. Med. - 2013. - Vol. 24. - P. 486-491.

7. Cataland S. R., Yang S., Wu H. M. The use of ADAMTS13 activity, platelet count and serum creatinine to differentiate acquired thrombotic thrombocytopenic purpura from other thrombotic microangiopathies // Br. J. Haematol. - 2012. Vol. 157. - P. 501-503.

8. Cataland S. R. Complement biomarkers and diagnosis of aHUS // Blood. 2014. - Vol. 123, № 24. - URL: ncbi.nlm.nih.gov

9. Chan M. R., Thomas C. P., Torrealba J. R. et al. Recurrent atypical hemolytic uremic syndrome associated with factor I mutation in a living related renal transplant recipient // Am. J. Kidney Dis. - 2009. - Vol. 53. - P. 321-326.

10. Constantinescu A. R., Bitzan M., Weiss L. S. et al. Non-enteropathic hemolytic uremic syndrome: causes and short-term course // Am. J. Kidney Dis. - 2004. Vol. 43. - P. 976-982

11. Coppo P., Schwarzinger M., Buffet M. et al. French Reference Center for Thrombotic Microangiopathies. Predictive features of severe acquired ADAMTS13 deficiency in idiopathic thrombotic microangiopathies: the French TMA reference center experience // PLoS One. - 2010. - Vol. 5. P. e10208.

12. Donne R. L, Abbs I Reccurence of hemolytic uremic syndrome after live related renal transplantation associated with subsequent de novo disease in the donor // Am. J. Kidney dis. - 2002. - URL: ncbi.nlm.nih.gov_ajkd.org

13. Dragon-Durey M. A., Sethi S. K., Bagga A. et al. Clinical features of anti-factor $\mathrm{H}$ autoantibody_associated hemolytic uremic syndrome // J. Am. Soc. Nephrol. 2010. - Vol. 21. - P. 2180-2187.

14. Fremeaux-Bacchi V., Fakhouri F., Garnier A. et al. Genetics and outcome of atypical hemolytic uremic syndrome: a nationwide French series comparing children and adults // Clin. J. Am. Soc. Nephrol. - 2013. - Vol. 8. - P. 554-562.

15. Gasser C., Gautier E., Steck A. et al. Hemolytic-uremic syndrome: bilateral necrosis of the renal cortex in acute acquired hemolytic anemia // Schweiz Med. Wochenschr. - 1955. - Vol. 85. - P. 905-909.

16. Greenbaum L. A., Fila M., Tsimaratos M. Eculizumab (ECU) inhibits thrombotic microangiopathy (TMA) and improves renal function in pediatric atypical hemolytic uremic syndrome (aHUS) patients (pts). Presented at American Society of Nephrology (ASN) Kidney Week 2013, Atlanta, Ga., November 9, 2013

17. Hofer J., Rosales A., Fischer C. et al. Extra-renal manifestation of complement-mediated thrombotic microangiopathies // Front. Pediatr. 2014. - Vol. 2. - P. 97.
18. Kavanagh D., Goodship T. H. Atypical hemolytic uremic syndrome, genetic basis and clinical manifesatations // Hematology Am. Soc. Hemat. Educ. Program. - 2011. - P. 15-17. - URL: ncbi.nlm.nih.gov

19. Keating G. M. Eculizumab: a review of its use in atypical haemolytic uraemic syndrome // Drugs. - 2013. - Vol. 73. - P. 2053-2066.

20. Keir L., Coward R. J. Advances in our understanding of the pathogenesis of glomerular thrombotic microangiopathy // Pediatr. Nephrol. - 2011. Vol. 26. - P. 523-533.

21. Kremer Hovinga J. A., Vesely S. K., Terrell D. R. et al. Survival and relapse in patients with thrombotic thrombocytopenic purpura // Blood. - 2010. Vol. 115. - P. 1500-1511.

22. Legendre C. M., Licht C., Muus P. et al. Terminal complement inhibitor eculizumab in atypical hemolytic-uremic syndrome // N. Engl. J. Med. - 2013. Vol. 368. - P. 2169-2181.

23. Loirat C., Freaux-Bacchi V. Atipical hemolytic-uremic syndrome // Orhanet. J. Rare Dis. - 2011. - Vol. 8. - P. 6-60.

24. Noris M., Caprioli J., Bresin E. et al. Relative role of genetic complement abnormalities in sporadic and familial aHUS and their impact on clinical phenotype // Clin. J. Am. Soc. Nephrol. - 2010. - Vol. 5. - P. 1844-1859.

25. Noris M., Galbusera M., Gastoldi S. et al. Dynamics of complement activation in aHUS and how to monitor eculizumab therapy // Blood. - 2014. - Vol. 124. P. 1715-1726.

26. Noris M., Remuzzi G. Atypical hemolytic-uremic syndrome // N. Engl. J. Med. 2009. - Vol. 361. - P. 1676-1687.

27. Noris M., Remuzzi G. Cardiovascular complications in atypical haemolytic uraemic syndrome // Nat. Rev. Nephrol. - 2014. - Vol. 10. - P. 174-180.

28. Pourrat O., Coudroy F. P. Differentiation between severe HELLP syndrome and trombotic microangiopathy, trombotic thrombocytopenic purpura and other imitators // European Journal of Obstetrics \& Gynecology and Reproductive Biology. - 2015. - Vol. 189. - P. 68-72.

29. Rafiq A. Atypical hemolytic-uremic syndrome // Am. J. Case Rep. - 2015. Vol. 16. - P. 109-114.

30. Saland J. Liver-kidney transplantation to cure atypical HUS: still an option post-eculizumab? // Pediatr. Nephrol. - 2014. - Vol. 29. - P. 329-332.

31. Sanches-Corral P., Melgosa M. Advanced in understanding aetiogy of atupical hemolytic uraemic syndrome // Br. J. Haemotol. - 2010. - Vol. 150. P. 529-542.

32. Scully M., Goodship T. How I treat thrombotic thrombocytopenic purpura and atypical haemolytic uraemic syndrome // Br. J. Haematol. - 2014. Vol. 164. - P. 759-766.

33. Sellier-Leclerc A. L., Fremeaux-Bacchi V., Dragon-Durey M. A. et al. French Society of Pediatric Nephrology. Differential impact of complement mutations on clinical characteristics in atypical hemolytic uremic syndrome // J. Am. Soc. Nephrol. - 2007. - Vol. 18. - P. 2392-2400.

34. Shrivastava M. Early diagnosis and management of postpartum hemolytic uremic syndrome with plasma exchange // Transfus. Apheresis Sci. - 2011. Vol. 44. - P. 257-262

35. Thompson R. A., Winterborn M. H. Hypocomplementaemia due to a genetic deficiency of beta $1 \mathrm{H}$ globulin // Clin. Exp. Immunol. - 1981. - Vol. 46. P. $110-119$.

36. Warwicker P., Goodship T. H., Donne R. L. et al. Genetic studies into inherited and sporadic hemolytic uremic syndrome // Kidney Int. - 1998. - Vol. 53. P. 836-844.

37. Wong E. K., Goodship T. H., Kavanagh D. Complement therapy in atypical haemolytic uraemic syndrome (aHUS) // Mol. Immunol. - 2013. - Vol. 56. P. 199-212.

38. Zuber J., Fakhouri F., Roumenina L. T. et al. French Study Group for aHUS/C3G. Use of eculizumab for atypical haemolytic uraemic syndrome and C3 glomerulopathies // Nat. Rev. Nephrol. - 2012. - Vol. 8. P. 643-657.

39. Zuber J., Le Quintrec M., Morris H. et al. Targeted strategies in the prevention and management of atypical HUS recurrence after kidney transplantation // Transplant Rev. (Orlando). - 2013. - Vol. 27. - P. 117-125.

\section{REFERENCES}

1. Clinical recommendations for diagnostics and treatment of atypical hemolytic-uremic syndrome. Nefrologiya i dializ. 2015, vol. 17, no. 3, http://www.nephro. $\mathrm{ru} /$ content/files/242-264.pdf 
2. Ariceta G., Besbas N., Johnson S. et al. European Paediatric Study Group for HUS. Guideline for the investigation and initial therapy of diarrhea-negative hemolytic uremic syndrome. Pediatr. Nephrol., 2009, vol. 24, pp. 687-696.

3. Besbas N., Karpman D., Landau D. et al. A classification of hemolytic uremic syndrome and thrombotic thrombocytopenic purpura and related disorders Kidney Int., 2006, vol. 70, pp. 423-431.

4. Campistol J.M., Arias M., Ariceta G. et al. An update for atypical haemolytic uraemic syndrome: diagnosis and treatment. A consensus document. Nefrologia, 2013, vol. 33, pp. 27-45.

5. Caprioli J., Noris M., Brioschi S. et al. International Registry of Recurrent and Familial HUS/TTP. Genetics of HUS: the impact of MCP, CFH, and IF mutations on clinical presentation, response to treatment, and outcome. Blood 2006, vol. 108, pp. 1267-1279.

6. Cataland S.R., Wu H.M. Atypical hemolytic uremic syndrome and thrombotic thrombocytopenic purpura: clinically differentiating the thrombotic microangiopathies. Eur. J. Intern. Med., 2013, vol. 24, pp. 486-491

7. Cataland S.R., Yang S., Wu H.M. The use of ADAMTS13 activity, platelet count, and serum creatinine to differentiate acquired thrombotic thrombocytopenic purpura from other thrombotic microangiopathies. Br. J. Haematol., 2012, vol. 157 , pp. 501-503

8. Cataland S.R. Complement biomarkers and diagnosis of aHUS. Blood 2014 vol. 123, no. 24, URL: ncbi.nlm.nih.gov

9. Chan M.R., Thomas C.P., Torrealba J.R. et al. Recurrent atypical hemolytic uremic syndrome associated with factor I mutation in a living related renal transplant recipient. Am. J. Kidney Dis., 2009, vol. 53, pp. 321-326.

10. Constantinescu A.R., Bitzan M., Weiss L.S. et al. Non-enteropathic hemolytic uremic syndrome: causes and short-term course. Am. J. Kidney Dis., 2004, vol. 43 , pp. $976-982$.

11. Coppo P., Schwarzinger M., Buffet M. et al. French Reference Center for Thrombotic Microangiopathies. Predictive features of severe acquired ADAMTS13 deficiency in idiopathic thrombotic microangiopathies: the French TMA reference center experience. PLoS One, 2010, vol. 5, pp. e10208.

12. Donne R.L., Abbs I. Reccurence of hemolytic uremic syndrome after live related renal transplantation associated with subsequent de novo disease in the donor. Am. J. Kidney Dis., 2002, URL: ncbi.nlm.nih.gov_ajkd.org

13. Dragon-Durey M.A., Sethi S.K., Bagga A. et al. Clinical features of anti-factor H autoantibody_associated hemolytic uremic syndrome. J. Am. Soc. Nephrol. 2010, vol. 21, pp. 2180-2187.

14. Fremeaux-Bacchi V., Fakhouri F., Garnier A. et al. Genetics and outcome of atypical hemolytic uremic syndrome: a nationwide French series comparing children and adults. Clin. J. Am. Soc. Nephrol., 2013, vol. 8, pp. 554-562.

15. Gasser C., Gautier E., Steck A. et al. Hemolytic-uremic syndrome: bilateral necrosis of the renal cortex in acute acquired hemolytic anemia. Schweiz Med. Wochenschr., 1955, vol. 85, pp. 905-909.

16. Greenbaum L.A., Fila M., Tsimaratos M. Eculizumab (ECU) inhibits thrombotic microangiopathy (TMA) and improves renal function in pediatric atypical hemolytic uremic syndrome (aHUS) patients (pts). Presented at American Society of Nephrology (ASN) Kidney Week 2013, Atlanta, Ga., November 9,2013

17. Hofer J., Rosales A., Fischer C. et al. Extra-renal manifestations of complement-mediated thrombotic microangiopathies. Front. Pediatr., 2014, vol. 2 pp. 97.

18. Kavanagh D., Goodship T.H. Atypical hemolytic uremic syndrome, genetic basis and clinical manifesatations. Hematology Am. Soc. Hemat. Educ. Program. 2011, pp. 15-17. URL: ncbi.nlm.nih.gov

19. Keating G.M. Eculizumab: a review of its use in atypical haemolytic uraemic syndrome. Drugs, 2013, vol. 73, pp. 2053-2066.

20. Keir L., Coward R.J. Advances in our understanding of the pathogenesis of glomerular thrombotic microangiopathy. Pediatr. Nephrol., 2011, vol. 26, pp. 523-533.

21. Kremer Hovinga J.A., Vesely S.K., Terrell D.R. et al. Survival and relapse in patients with thrombotic thrombocytopenic purpura. Blood, 2010, vol. 115 pp. 1500-1511.

22. Legendre C.M., Licht C., Muus P. et al. Terminal complement inhibitor eculizumab in atypical hemolytic-uremic syndrome. N. Engl. J. Med., 2013, vol. 368, pp. 2169-2181.

23. Loirat C., Freaux-Bacchi V. Atipical hemolytic-uremic syndrome. Orhanet. J. Rare Dis., 2011, vol. 8, pp. 6-60.
24. Noris M., Caprioli J., Bresin E. et al. Relative role of genetic complement abnormalities in sporadic and familial aHUS and their impact on clinical phenotype. Clin. J. Am. Soc. Nephrol., 2010, vol. 5, pp. 1844-1859.

25. Noris M., Galbusera M., Gastoldi S. et al. Dynamics of complement activation in aHUS and how to monitor eculizumab therapy. Blood, 2014, vol. 124, pp. 1715-1726.

26. Noris M., Remuzzi G. Atypical hemolytic-uremic syndrome. N. Engl. J. Med., 2009, vol. 361, pp. 1676-1687.

27. Noris M., Remuzzi G. Cardiovascular complications in atypical haemolytic uraemic syndrome. Nat. Rev. Nephrol., 2014, vol. 10, pp. 174-180.

28. Pourrat O., Coudroy F.P. Differentiation between severe HELLP syndrome and trombotic microangiopathy, trombotic thrombocytopenic purpura and other imitators. European Journal of Obstetrics \& Gynecology and Reproductive Biology, 2015, vol. 189, pp. 68-72.

29. Rafiq A. Atypical hemolytic-uremic syndrome. Am. J. Case Rep., 2015, vol. 16, pp. 109-114.

30. Saland J. Liver-kidney transplantation to cure atypical HUS: still an option post-eculizumab? Pediatr. Nephrol., 2014, vol. 29, pp. 329-332.

31. Sanches-Corral P., Melgosa M. Advanced in understanding aetiogy of atupical hemolytic uraemic syndrome. Br. J. Haemotol., 2010, vol. 150, pp. 529-542.

32. Scully M., Goodship T. How I treat thrombotic thrombocytopenic purpura and atypical haemolytic uraemic syndrome. Br. J. Haematol., 2014, vol. 164, pp. 759-766.

33. Sellier-Leclerc A.L., Fremeaux-Bacchi V., Dragon-Durey M.A. et al. French Society of Pediatric Nephrology. Differential impact of complement mutations on clinical characteristics in atypical hemolytic uremic syndrome. J. Am. Soc. Nephrol., 2007, vol. 18, pp. 2392-2400.

34. Shrivastava M. Early diagnosis and management of postpartum hemolytic uremic syndrome with plasma exchange. Transfus. Apheresis Sci., 2011, vol. 44, pp. 257-262.

35. Thompson R.A., Winterborn M.H. Hypocomplementaemia due to a genetic deficiency of beta $1 \mathrm{H}$ globulin. Clin. Exp. Immunol., 1981, vol. 46, pp. 110-119.

36. Warwicker P., Goodship T.H., Donne R.L. et al. Genetic studies into inherited and sporadic hemolytic uremic syndrome. Kidney Int., 1998, vol. 53, pp. 836-844.

37. Wong E.K., Goodship T.H., Kavanagh D. Complement therapy in atypical haemolytic uraemic syndrome (aHUS). Mol. Immunol., 2013, vol. 56, pp. 199-212.

38. Zuber J., Fakhouri F., Roumenina L.T. et al. French Study Group for aHUS/C3G. Use of eculizumab for atypical haemolytic uraemic syndrome and C3 glomerulopathies. Nat. Rev. Nephrol., 2012, vol. 8, pp. 643-657.

39. Zuber J., Le Quintrec M., Morris H. et al. Targeted strategies in the prevention and management of atypical HUS recurrence after kidney transplantation. Transplant Rev. (Orlando), 2013, vol. 27, pp. 117-125.

\section{ДЛЯ КОРРЕСПОНДЕНЦИИ:}

ФГБУ «НЦ акушерства, гинекологии и перинатологии им. В. И. Кулакова» МЗ РФ, 117997, г. Москва, ул. Академика Опарина, д. 4.

\section{Пьюрегов Алексей Викторович}

доктор медицинских наук, заведующий отделением анестезиологии-реанимации.

Тел.: 8 (495) 438-33-10.

E-mail:pyregov@mail.ru

\section{Федорова Татьяна Анатольевна}

доктор медицинских наук, профессор, заведующая отделом трансфузиологии и экстракорпоральной гемокоррекции.

\section{Королев Алексей Юрвевич}

врач отделения анестезиологии-реанимации.

\section{Гришук Константин Иванович}

врач отделения анестезиологии-реанимации. 


\section{FOR CORRESPONDENCE:}

Research Center for Obstetrics, Gynecology and Perinatology, Russian Ministry of Health,

4, Akademika Oparina St,, Moscow, 117997.

\section{Alexey V. Pyregov}

Doctor of Medical Sciences, Head of Anesthesiology and Intensive Care Department.

Phone: +7 (495) 438-33-10.

E-mail:pyregov@mail.ru

\section{Tatiana A. Fedorova}

Doctor of Medical Sciences, Head of Transfusiology and Extracorporal Hemocorrection Department.

\section{Alexey Yu. Korolev}

Doctor of Anesthesiology and Intensive Care Department.

\section{Konstantin I. Grischuk}

Doctor of Anesthesiology and Intensive Care Department. 